\title{
Adiponectin inhibits D-gal-induced cardiomyocyte senescence via AdipoR1/APPL1
}

\author{
RUIYING LIU ${ }^{1}$, JING MENG ${ }^{2}$ and DANFEI LOU ${ }^{2}$ \\ ${ }^{1}$ Department of Geriatric Cardiovascular, General Hospital of Southern Theater Command, Chinese People's Liberation Army, \\ Guangzhou, Guangdong 510010; ${ }^{2}$ Department of Geriatrics, Shanghai Municipal Hospital of Traditional Chinese Medicine, \\ Shanghai University of Traditional Chinese Medicine, Shanghai 200071, P.R. China
}

Received December 17, 2020; Accepted April 29, 2021

DOI: $10.3892 / \mathrm{mmr} .2021 .12358$

\begin{abstract}
The aim of the present study was to examine whether adiponectin could inhibit cardiomyocyte senescence induced by D-galactose (D-gal), and whether it functioned via the adiponectin receptor 1 (AdipoR1)/adaptor protein phosphotyrosine interacting with $\mathrm{PH}$ domain and leucine zipper 1 (APPL1) signaling pathway. For this purpose, the expression levels of adiponectin, AdipoR1 and APPL1 in mouse plasma and myocardial tissues were detected via reverse transcription-quantitative PCR (RT-qPCR) and western blotting. An adiponectin-overexpression plasmid was transfected into D-gal-treated H9c2 cells prior to the detection of AdipoR1 and APPL1 expression by RT-qPCR. Senescence-associated $\beta$-galactose staining was then performed to observe cellular senescence following the transfection of small interfering RNAs (si) targeting AdipoR1 and APPL1 into D-gal-treated $\mathrm{H} 9 \mathrm{c} 2$ cells overexpressing adiponectin. Commercial kits were used to detect reactive oxygen species (ROS) production and malondialdehyde (MDA) content in the different groups. The expression levels of heme oxygenase (HO)-1 and high mobility group box 1 (HMGB1) were examined by western blot analysis. The results revealed that the expression levels of adiponectin, AdipoR1 and APPL1 were downregulated in aged mouse plasma, myocardial tissues and D-gal-treated cardiomyocytes. It was also observed that AdipoR1 and APPL1 expression levels were significantly upregulated following the overexpression of adiponectin into D-gal-treated cardiomyocytes. Moreover, adiponectin overexpression reduced cellular senescence induced by D-gal and the expression of p16 and $\mathrm{p} 21$; these effects were reversed following transfection with
\end{abstract}

Correspondence to: Dr Danfei Lou, Department of Geriatrics, Shanghai Municipal Hospital of Traditional Chinese Medicine, Shanghai University of Traditional Chinese Medicine, 274 Middle Zhijiang Road, Jing'an, Shanghai 200071, P.R. China

E-mail: loudanfeildf@163.com

Key words: adiponectin, cardiomyocyte senescence, adiponectin receptor 1, adaptor protein, phosphotyrosine interacting with $\mathrm{PH}$ domain and leucine zipper 1
si-AdipoR1 and si-APPL1. Adiponectin also downregulated the levels of ROS and MDA in D-gal-treated H9c2 cells via AdipoR1/APPL1. Additionally, the release of HO-11/HMGB1 was affected by adiponectin via AdipoR1/APPL1, and adiponectin/AdipoR1/APPL1 suppressed ROS production via HO-1/HMGB1. On the whole, the present study demonstrated that adiponectin played an inhibitory role in cardiomyocyte senescence via the AdioR1/APPL1 signaling pathway and inhibited the levels of oxidative stress in senescent cardiomyocytes via the HO-1/HMGB1 signaling pathway.

\section{Introduction}

Population aging has become a common issue worldwide; it is estimated that individuals aged $\geq 60$ years will constitute $\geq 20 \%$ of the world's total population by 2050 (1). The challenges associated with an aging population already pose great difficulties; however, it is estimated that in the coming years, an increasing number of countries will be faced with graver public health concerns and heavier healthcare burdens, of which cardiovascular disease (CVD) will account for a large percentage (2).

CVD, known for its high morbidity and mortality, is caused by a number of factors, among which intrinsic aging plays a prominent role; it is also the leading cause of mortality for seniors of $>65$ years of age (3). Oxidative stress is considered to be a crucial factor that triggers heart aging. A growing body of evidence has confirmed that reactive oxygen species (ROS) are produced in increasing amounts in myocardial tissues as age increases (4). Therefore, the effective inhibition of ROS is expected to attenuate heart aging, thus relieving its detrimental effects on human health (5). D-galactose (D-gal) treatment has been used in a number of rodent models of aging $(6,7)$, including brain and heart aging, and has been reported to notably induce aging-related changes, such as reducing thymus coefficients and increasing pathological injury and cellular senescence in the liver, spleen and hippocampus (8). D-gal is a reducing sugar that, when it accumulates in the body, reacts with free amines from amino acids in proteins and peptides to form an unstable compound Schiff base, which persists for several months after as the compound is oxidized to very stable advanced glycation end products (AGEs) (9). AGEs increase during aging and are considered to be one of the signs of aging (10). 
Adiponectin, an adipocyte-specific hormone derived from fat, performs an essential anti-inflammatory and antioxidant function in CVDs (11). A previous study indicated that the transfection of adiponectin into endothelial progenitor cells protected the cognitive function of rats with D-gal-induced aging (12). However, at present, detailed research on the effectiveness and mechanisms of adiponectin in the aging of myocardial fibers, namely cardiomyocyte senescence, is limited. Adiponectin has two cell surface homologous receptors, adiponectin receptor 1 (AdipoR1) and AdipoR2, both of which are expressed in various types of tissues (13). In addition, adaptor protein phosphotyrosine interacting with $\mathrm{PH}$ domain and leucine zipper 1 (APPL1), as an adapter protein, has been identified as the signal transductor of AdipoR1/2 (14).

The current state of the aging population and the previous research findings prompted further investigations into the association between adiponectin and heart aging induced by D-gal, in addition to determining whether adiponectin exerts an inhibitory effect on cardiomyocyte senescence through the AdipoR1/APPL1 signaling pathway. The present study aimed to provide an innovative approach that may be used to attenuate heart aging. The findings presented herein may provide novel insight into the treatment of CVDs.

\section{Materials and methods}

Animals and treatments. BALB/c mice $(\mathrm{n}=16$; age, 2 and 15 months old; weight, $25 \mathrm{~g}$-30 g; male) were purchased from the Guangdong Medical Laboratory Animal Center and housed in the following two groups (eight mice per group): Young mice ( 2 months old) and aged mice ( 15 months old). The mice were housed in a controlled environment at a temperature of $20-25^{\circ} \mathrm{C}$ and a humidity level of $50-70 \%$ under a 12-h light/dark cycle and were provided with food and water ad libitum. Following acclimation to the laboratory environment, all 16 mice were sacrificed by cervical dislocation following anesthesia with $350 \mathrm{mg} / \mathrm{kg}$ chloral hydrate $(10 \%$, abdominal injection). Mice were checked for complete cardiac arrest and pupil dilation to confirm death and then their blood samples and myocardial tissue samples were collected for subsequent analysis. All procedures related to animal experiments in the present study were approved by the Shanghai Municipal Hospital of Traditional Chinese Medicine (approval no. dw2019018; Shanghai, China).

Western blot analysis. Total proteins were extracted from the tissues using RIPA lysis buffer (Beyotime Institute of Biotechnology) and protein concentration was determined using BCA kits (Beyotime Institute of Biotechnology) according to the manufacturer's protocol. SDS-PAGE loading buffer (Beyotime Institute of Biotechnology) was added to the protein samples, which were then boiled in a water bath for 3-5 min to achieve protein denaturation. Subsequently, $40 \mu \mathrm{g}$ protein/lane was electrophoresed with $12 \%$ SDS-PAGErunning buffer (Beyotime Institute of Biotechnology) at room temperature, and then transferred to a PVDF membrane (Thermo Fisher Scientific, Inc.), which was blocked in 5\% skimmed milk on a shaker (Beyotime Institute of Biotechnology) for $1 \mathrm{~h}$ at room temperature. TBS with Tween-20 (0.2\%, TBST) buffer (Shanghai Aladdin Bio-Chem Technology Co., Ltd.) was then used to wash the membrane three times for $1 \mathrm{~min}$ each time, prior to incubation with the primary antibody dilution buffer (Nanjing Channel Technology Group) overnight at $4^{\circ} \mathrm{C}$. On the second day, TBST buffer was again used to wash the membrane three times for 5 min each time, followed by incubation with the secondary antibody dilution buffer (Nanjing Channel Technology Group) at room temperature for $2 \mathrm{~h}$. Finally, the membrane was washed three times with TBST for $5 \mathrm{~min}$ each time prior to chemiluminescence detection (ECL Western substrate; Thermo Fisher Scientific, Inc.) using Image Lab software (Version 4.0; Bio-Rad Laboratories, Inc.). GAPDH (1:1,000; cat. no. 5174; Cell Signaling Technology, Inc.) was selected as the internal control. All experiments were performed in triplicate. The antibodies were as follows: Anti-p16 (1:1,000; cat. no. 18769; Cell Signaling Technology, Inc.), anti-p21 (1:1,000; cat. no. 2947; Cell Signaling Technology, Inc.), anti-adiponectin (1:1,000; cat. no. 2789; Cell Signaling Technology, Inc.), anti-AdipoR1 (1:800; cat. no. bs-0610R; BIOSS), anti-APPL1 (1:1,000; cat. no. 3858; Cell Signaling Technology, Inc.), anti-heme oxygenase (HO)-1 (1:1,000; cat. no. 43966; Cell Signaling Technology, Inc.), anti-high mobility group box 1 (HMGB1; 1:1,000; cat. no. 3935; Cell Signaling Technology, Inc.) and mouse anti-rabbit secondary antibody (1:1,000; cat. no. 5127; Cell Signaling Technology, Inc.).

RNA extraction and reverse transcription-quantitative $P C R$ (RT-qPCR). Total RNA was isolated from the mouse blood samples using TRIzol ${ }^{\circledR}$ reagent (Invitrogen; Thermo Fisher Scientific, Inc.) according to the manufacturer's protocol. The RNA purity was examined using a NanoDrop 2000 spectrophotometer (Thermo Fisher Scientific, Inc.). Total RNA $(2.5 \mu \mathrm{g})$ was reverse transcribed into cDNA using an EasyScript ${ }^{\circledR}$ First-Strand cDNA Synthesis SuperMix (TransGen Biotech Co., Ltd.) according to the manufacturer's instructions. qPCR was performed on a Veriti ${ }^{\mathrm{TM}}$ 96-Well Thermal Cycler (Thermo Fisher Scientific, Inc.) with the following thermocycling conditions: Initial denaturation at $85^{\circ} \mathrm{C}$ for $30 \mathrm{sec}$, followed by 22 cycles at $55^{\circ} \mathrm{C}$ for $30 \mathrm{sec}$ and $72^{\circ} \mathrm{C}$ for $30 \mathrm{sec}$. A mixture of $10 \mu \mathrm{l} \mathrm{SYBR}{ }^{\mathrm{TM}}$ Green PCR Master Mix (Thermo Fisher Scientific, Inc.), $7 \mu 1$ water and $1 \mu \mathrm{l}$ primer working solution (Shanghai GenePharma Co., Ltd.) was added to each well containing $2 \mu \mathrm{l}$ cDNA for each reaction. Each sample was tested in triplicate. The plate was then centrifuged $(8,000 \mathrm{x} \mathrm{g}, 20 \mathrm{~min})$ to spin down the solution on a Centrifuge 5810 device (Eppendorf) at $4^{\circ} \mathrm{C}$. GAPDH was used as the internal reference gene. The analysis of the data was performed using the $2^{-\Delta \Delta \mathrm{Cq}}$ method (15). The sequence of primer pairs were as follows: Adiponectin forward (F), 5'-GCA TTCAGTGTGGGATTGGAG-3' and reverse (R), 5'-AGACTG TGATGTGGTAGGCAAAG-3'; AdipoR1 F, 5'-CAAGGC TGAAGAAGAACAAGC-3' and R, 5'-AAGGAGGGCATA GGTGGTCT-3'; APPL1 F, 5'-AGCCAGTGACCCTTTATA TCTGC-3' and R, 5'-AGGTATCCAGCCTTTCGGGTT-3'; and GAPDH F, 5'-CTCTCTGCTCCTCCCTGTTC-3' and R, 5'-CGATACGGCCAAATCCGTTC-3'.

Cell culture and treatments. $\mathrm{H} 9 \mathrm{c} 2$ rat cardiomyocytes were obtained from The Cell Bank of Type Culture Collection of The Chinese Academy of Sciences and cultured in DMEM 
(Gibco; Thermo Fisher Scientific, Inc.) supplemented with 10\% high-quality FBS (Gibco; Thermo Fisher Scientific, Inc.), $100 \mathrm{U} / \mathrm{ml}$ penicillin and $100 \mathrm{mg} / \mathrm{ml}$ streptomycin; the medium was replaced every 2 days. Cells were maintained in a humidified incubator of $95 \%$ air and $5 \% \mathrm{CO}_{2}$ at $37^{\circ} \mathrm{C}$. Upon reaching $80 \%$ confluence, the $\mathrm{H} 9 \mathrm{c} 2$ cells were seeded into 96-well plates at a density of $2 \times 10^{3}$ cells/well and treated with 2.5, 5 and $10 \mathrm{~g} / \mathrm{l} \mathrm{D-gal} \mathrm{(Macklin,} \mathrm{Inc.)} \mathrm{at} 37^{\circ} \mathrm{C}$ for $24 \mathrm{~h}$ before being harvested for use in further experiments and analysis. Subsequently, When the density of H9c2 cells reached $\sim 70 \%$, overexpression plasmids for adiponectin (Ov-Adiponectin) were constructed (Shanghai GenePharma Co., Ltd.) and were transfected into H9c2 cells $(1.5 \mu \mathrm{g} /$ well $)$ using Lipofectamine ${ }^{\circledR}$ 3000 reagent (Invitrogen; Thermo Fisher Scientific, Inc.). The untransfected H9c2 cells induced by $10 \mathrm{~g} / \mathrm{l} \mathrm{D}$-gal were used as the negative control (NC) group for the Ov-Adiponectin group. Subsequently, small interfering RNAs (siRNAs/si) targeting AdipoR1 (siAdipoR1, 5'-AAGGTACTACTCAACTAGAAT GT-3') and APPL1 (siAPPL1, 5'-ATGATAGGATGTGAGATA AGTCC-3') were constructed (Shanghai GenePharma Co., Ltd.) and were transfected into Ov-Adiponectin $\mathrm{H} 9 \mathrm{c} 2$ cells using Lipofectamine 3000 reagent (Invitrogen; Thermo Fisher Scientific, Inc.), and negative control (si-NC; cat. no. 12935300) were obtained from Thermo Fisher Scientific, Inc. The D-gal-induced cells transfected with Ov-Adiponectin plasmid were defined as the NC group for both the D-gal $10 \mathrm{~g} / \mathrm{l}+\mathrm{Ov}$ Adiponectin + si-AdipoR1 and D-gal $10 \mathrm{~g} / \mathrm{l}+\mathrm{Ov}$-Adiponecti $\mathrm{n}+\mathrm{si}$-APPL1 groups. The plasmids were transfected into cells at a concentration of $50 \mathrm{ng} / \mathrm{ml}$. After $12 \mathrm{~h}$ incubation at $37^{\circ} \mathrm{C}$, the medium was replaced with fresh DMEM and cells were cultured for $72 \mathrm{~h}$ at $37^{\circ} \mathrm{C}$ before subsequent experiments.

Senescence-associated $\beta$-galactose (SA- $\beta$-gal) staining. SA- $\beta$-gal staining was performed using the Senescence Assay kit (cat. no. ab65351; Abcam) to observe signs of aging in H9c2 cells induced by $\mathrm{D}$-gal, according to the manufacturer's protocol. Briefly, H9c2 cells treated with D-gal were washed with PBS and fixed with fixative solution for $10 \mathrm{~min}$ at room temperature, after which the cells were washed with PBS again and incubated in a staining solution mix for $1 \mathrm{~h}$ at $37^{\circ} \mathrm{C}$. The solutions and staining supplement mentioned were all included with the kit. Finally, seven representative images (magnification, x100) were obtained using a fluorescence microscope (Leica Microsystems $\mathrm{GmbH}$ ) from randomly selected fields of view. The procedures were performed in triplicate for each group.

ROS level detection. A ROS Assay kit (cat. no. S0033S, Beyotime Institute of Biotechnology) was used to examine the production of ROS in $\mathrm{H} 9 \mathrm{c} 2$ cells. Zinc protoporphyrin (ZnPP; $100 \mu \mathrm{g} / \mathrm{ml}$; Sigma-Aldrich; Merck KGAA, an inhibitor of HO-1, was co-incubated with cells at $37^{\circ} \mathrm{C}$ for $20 \mathrm{~min}$. Cells at a density of $1 \times 10^{6}$ cells $/ \mathrm{ml}$ were collected and suspended in diluted 2'-7'Dichlorofluorescin diacetate (DCFH-DA) at a concentration of $10 \mu \mathrm{mol} / 1$. Following incubation for $20 \mathrm{~min}$ at $37^{\circ} \mathrm{C}$, the cells were washed three times with serum-free cell culture medium to sufficiently remove the DCFH-DA that did not enter the cells. Finally, an excitation wavelength of $488 \mathrm{~nm}$ and emission wavelength of $525 \mathrm{~nm}$ were applied using a microplate reader to detect the intensity of fluorescence before and after stimulation in real-time or at different time points.
Malondialdehyde (MDA) content detection. The lipid peroxidation levels of $\mathrm{H} 9 \mathrm{c} 2$ cells subjected to the different treatments were examined using a Lipid Peroxidation MDA Assay kit (cat. no. S0131S, Beyotime Institute of Biotechnology). The preparation of TBA stock solution and MDA working solution, as well as the dilution of the standard substances were all performed in accordance with the product manual. The MDA content in the sample solution was calculated using a standard curve. The experiment was replicated in triplicate.

Statistical analysis. Data are presented as the mean \pm SD. Data were analyzed using GraphPad Prism 8.0.1 software (GraphPad Software, Inc.). Statistical differences were determined using a one-way ANOVA followed by Tukey's post hoc test for group comparisons. $\mathrm{P}<0.05$ was considered to indicate a statistically significant difference.

\section{Results}

Adiponectin, AdipoR1 and APPL1 expression levels are downregulated in aged mouse plasma and myocardial tissues. Western blot analysis was first performed to analyze the expression levels of the senescence-related genes, p16 and p21, in the myocardial tissues of both young mice and aged mice. A markedly higher expression of p16 and p21 was found in the tissues of the older group compared with the younger group (Fig. 1A). Furthermore, to explore the association between adiponectin and heart aging, the expression levels of adiponectin, AdipoR1 and APPL1 in the plasma of both the young and aged mice were analyzed by RT-qPCR, followed by the detection of these levels in the mouse myocardial tissues by western blot analysis. The results revealed that the relative mRNA and protein expression levels of adiponectin, AdipoR1 and APPL1 were downregulated in the older group compared with the younger group in both the plasma and myocardial tissues (Fig. 1B and C). These findings suggested a negative association between adiponectin expression and heart aging.

Expression levels of adiponectin, AdipoR1 and APPL1 are downregulated in D-gal-treated cardiomyocytes. To establish the cardiomyocyte model of senescence, western blot analysis was performed to detect the expression levels of p16 and p21 in $\mathrm{H} 9 \mathrm{c} 2$ cardiomyocytes treated with $2.5,5$ and $10 \mathrm{~g} / \mathrm{l} \mathrm{D}$-gal. The results revealed that, compared with the control group, the relative expression levels of p16 and p21 were elevated in a concentration-dependent manner in the groups with D-gal induction, and cells treated with $10 \mathrm{~g} / \mathrm{l} \mathrm{D}$-gal exhibited the most prominent elevation of p16 and p21 expression (Fig. 2A). Therefore, D-gal at the concentration of $10 \mathrm{~g} / \mathrm{l}$ was selected for use in subsequent experiments.

Adiponectin overexpression promotes the expression of AdipoR1 and APPL1 in D-gal-treated cardiomyocytes. To investigate the association between adiponectin and AdipoR1/APPL1 in senescent cardiomyocytes, RT-qPCR was performed to determine the expression of adiponectin following the transfection of adiponectin overexpression plasmid into D-gal-treated H9c2 cells. As shown in Fig. 2B-D, downregulated mRNA and protein expression levels of adiponectin, AdipoR1 and APPL1 were observed in the cells 

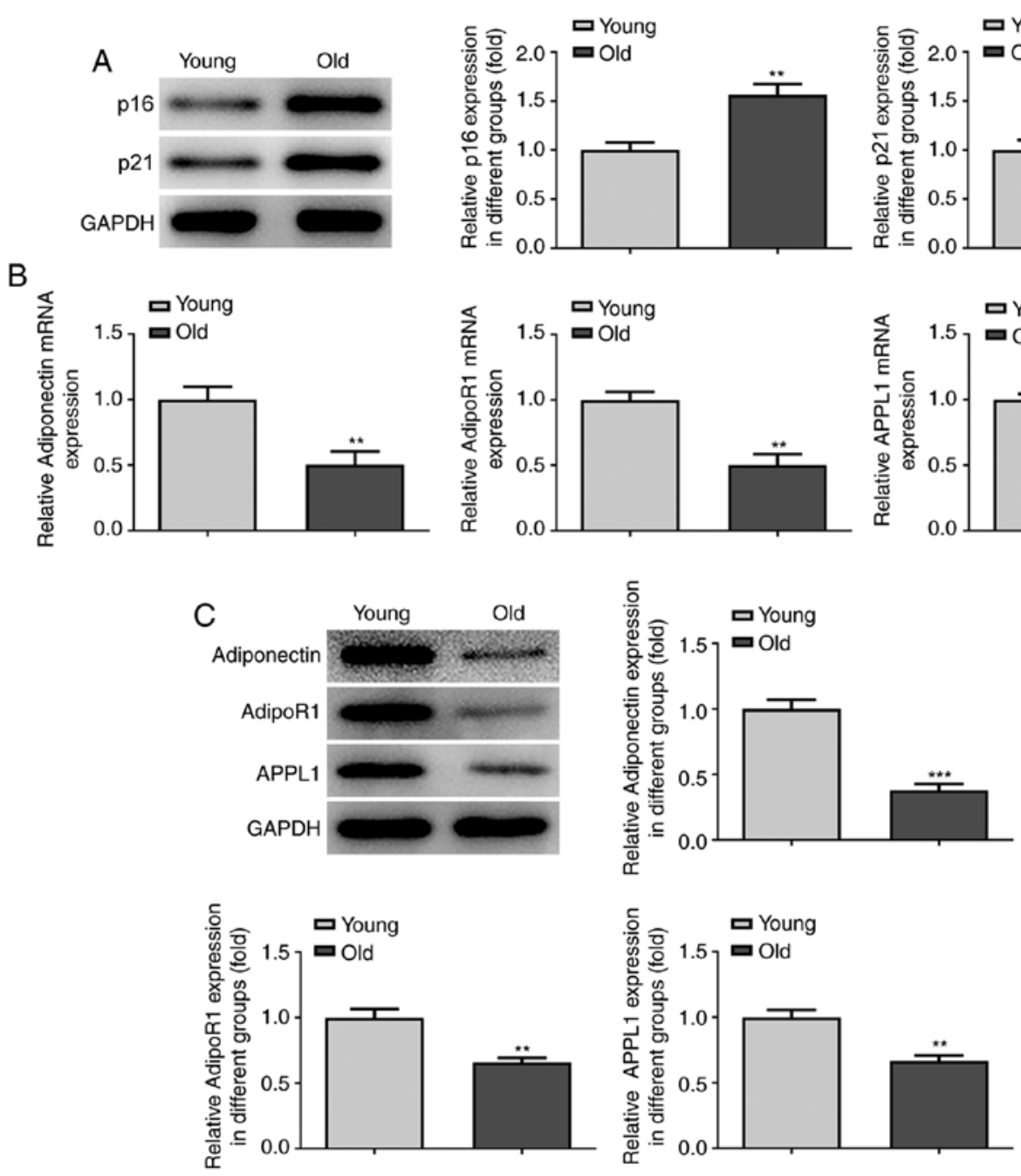

Figure 1. Expression of adiponectin, AdipoR1 and APPL1 is downregulated in aged mouse plasma and myocardial tissue. (A) Relative expression levels of p16 and p21 in myocardial tissues of young mice (aged 2 months) and aged mice (aged 15 months) were detected by western blotting. (B) Relative mRNA expression levels of adiponectin, AdipoR1 and APPL1 in the plasma of young mice and aged mice were detected by reverse transcription-quantitative PCR. (C) Relative expression levels of adiponectin, AdipoR1 and APPL1 in myocardial tissues of young mice and aged mice were analyzed using western blot analysis. ${ }^{* *} \mathrm{P}<0.01$, ${ }_{* * * *} \mathrm{P}<0.001$ vs. young mice. AdipoR1, adiponectin receptor 1; APPL1, adaptor protein phosphotyrosine interacting with PH domain and leucine zipper 1 .

treated with $10 \mathrm{~g} / \mathrm{l} \mathrm{D}$-gal compared with the control; these levels were markedly increased following the overexpression of adiponectin compared with the NC group, suggesting a promoting effect of adiponectin overexpression on AdipoR1/ APPL1 expression in D-gal-treated cardiomyocytes.

Adiponectin inhibits D-gal-induced cardiomyocyte senescence via AdipoR1/APPL1. To elucidate the mechanisms through which adiponectin affects cardiomyocyte senescence and whether its effects are through the AdipoR1/APPL1 signaling pathway, siRNAs targeting AdiopoR1 and APPL1 were constructed and transfected into H9c2 cells overexpressing adiponectin (Fig. S1A). The transfection efficiency was examined by RT-qPCR and western blotting, which demonstrated that the relative mRNA and protein expression levels of AdipoR1 and APPL1 were notably downregulated in the OV-adiponectin cells transfected with the siRNAs compared with the NC group (Fig. 3A-D). In addition, siRNA transfection in OV-adiponectin cells without D-gal treatment also exhibited downregulated mRNA and protein expression levels of AdipoR1 and APPL1 (Fig. S1B and C). It was further observed by SA- $\beta$-gal staining that transfection with OV-adiponectin significantly inhibited D-gal-induced cell senescence, which was, however, reversed to a certain degree by transfection with si-AdiopoR1 and si-APPL1 (Fig. 3E). Additionally, western blot analysis revealed that the expression levels of senescence-related proteins, p16 and $\mathrm{p} 21$, which were downregulated by adiponectin overexpression, were partially increased by transfection with si-AdiopoR 1 and si-APPL1 in the D-gal-treated H9c2 cells (Fig. 3F). Taken together, these results indicated an inhibitory effect of adiponectin on D-gal-induced cardiomyocyte senescence via regulating AdipoR1/APPL1.

Adiponectin inhibits the oxidative stress levels in D-gal-treated cardiomyocytes via AdipoR1/APPL1. ROS production was detected in D-gal-treated $\mathrm{H} 9 \mathrm{c} 2$ cells using commercial kits for a further exploration of the mechanisms through which adiponectin inhibits cardiomyocyte senescence. As shown in Fig. 4A, the relative ROS levels in the D-gal-treated $\mathrm{H} 9 \mathrm{c} 2$ cells were significantly decreased by transfection with OV-adiponectin, whereas they were increased by transfection with si-AdipoR1 and si-APPL1 (Fig. 4A and B). Furthermore, an MDA assay 
A

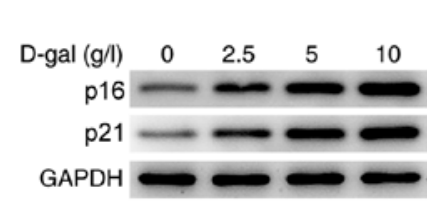

B

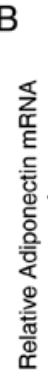

$$
\text { D-gal } 10 \mathrm{~g} / \mathrm{l}
$$

D-gal $10 \mathrm{~g} / \mathrm{l}+\mathrm{Ov}-\mathrm{NC}$

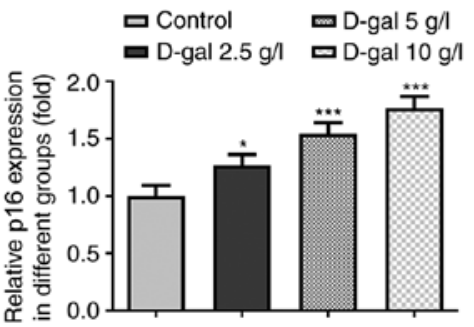

C

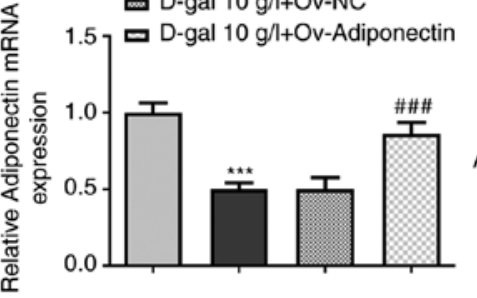

D

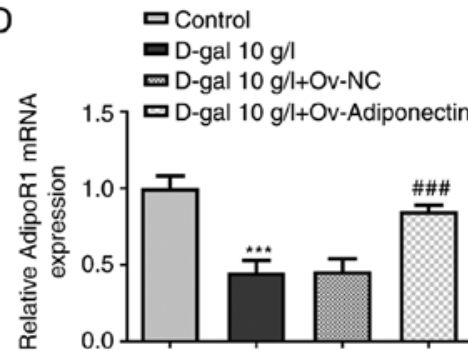

Adiponectin
II D-gal 10

III D-gal $10 \mathrm{~g} / \mathrm{l}+\mathrm{OV}-\mathrm{NC}$

IV D-gal $10 \mathrm{~g} / \mathrm{l}+\mathrm{O}$-adiponectin

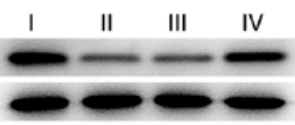

GAPDH

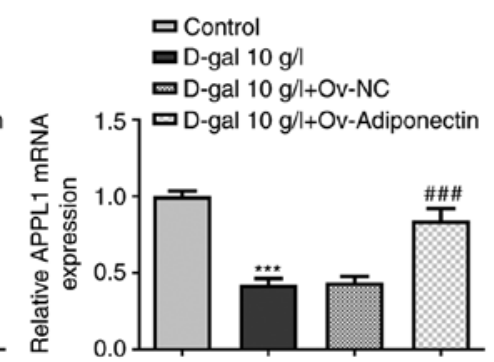

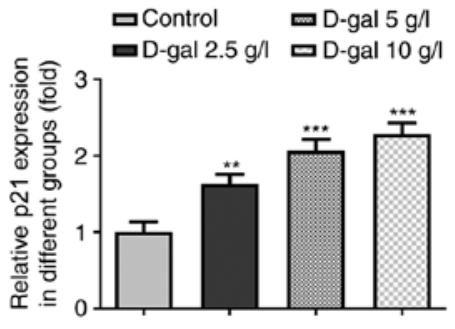

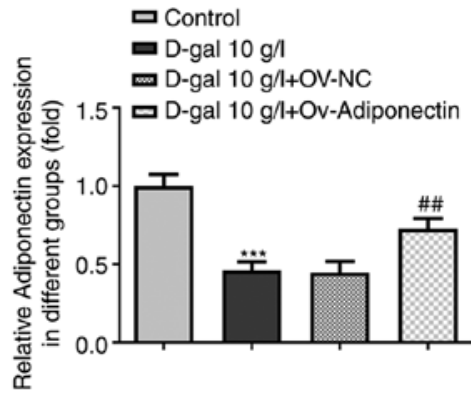

Figure 2. Expression of adiponectin, AdipoR1 and APPL1 is downregulated in D-gal-treated cardiomyocytes. (A) Relative expression levels of p16 and p21 in $\mathrm{H} 9 \mathrm{c} 2$ cells treated with $2.5,5$ and $10 \mathrm{~g} / 1$ of $\mathrm{D}$-gal were analyzed using western blot analysis. ${ }^{*} \mathrm{P}<0.05,{ }^{* *} \mathrm{P}<0.01,{ }^{* * *} \mathrm{P}<0.001$ vs. control. Transfection with Ov-adiponectin promotes the expression of AdipoR1 and APPL1 in D-gal-treated cardiomyocytes. (B) Relative mRNA expression of adiponectin in H9c2 cells before and after transfection with Ov-adiponectin plasmid was analyzed via RT-qPCR. ${ }^{* * *} \mathrm{P}<0.001$ vs. control; ${ }^{\# \#} \mathrm{P}<0.001 \mathrm{vs}$. D-gal $+\mathrm{Ov}-\mathrm{NC}$. (C) Relative protein expression of adiponectin in $\mathrm{H} 9 \mathrm{c} 2$ cells before and after transfection with $\mathrm{Ov}$-adiponectin plasmid was analyzed using RT-qPCR. ${ }^{* * *} \mathrm{P}<0.001$ vs. control; ${ }^{\# \#} \mathrm{P}<0.01$ vs. D-gal + Ov-NC. (D) Relative mRNA expression levels of AdipoR1 and APPL1 in H9c2 cells before and after transfection with Ov-adiponectin plasmids were detected using RT-qPCR. ${ }^{* * *} \mathrm{P}<0.001$ vs. control; ${ }^{\# \#} \mathrm{P}<0.001$ vs. D-gal + Ov-NC. AdipoR1, adiponectin receptor 1 ; APPL1, adaptor protein phosphotyrosine interacting with PH domain and leucine zipper 1; RT-qPCR, reverse transcription-quantitative PCR; NC, negative control; D-gal, D-galactose; Ov-, overexpression.

kit was utilized to detect the lipid peroxidation levels in $\mathrm{H} 9 \mathrm{c} 2$ cells; it was observed that adiponectin overexpression markedly reduced relative MDA expression in the D-gal treated H9c2 cells, whereas this was enhanced following transfection with si-AdipoR1 and si-APPL1 compared with the NC group (Fig. 4C). These results collectively suggested that adiponectin decreased the levels of oxidative stress in D-gal-induced cellular senescence through the AdipoR1/APPL1 signaling pathway.

Adiponectin affects the release of HO-1/HMGB1 via AdipoR I/ APPL1. To determine whether HO-1/HMGB1, an oxidative stress response-related pathway in the body (16), plays a role in the mechanisms of action of adiponectin in cardiomyocyte senescence, the expression levels of HO-1 and HMGB1 in the different groups were detected by western blot analysis. It was observed that the relative expression levels of HO-1 were significantly upregulated in the D-gal-treated $\mathrm{H} 9 \mathrm{c} 2$ cells following adiponectin overexpression, while following transfection with si-AdipoR1 and si-APPL1, HO-1 expression was decreased to a certain degree compared with the NC group (Fig. 5A). On the other hand, the relative expression of HMGB1 in D-gal-treated $\mathrm{H} 9 \mathrm{c} 2$ cells was decreased by adiponectin overexpression, and was significantly increased by transfection with si-AdipoR1 and si-APPL1 compared with the NC group (Fig. 5A). These findings suggested that adiponectin promotes HO-1 expression, while it inhibits HMGB1 expression through the AdioR1/ APPL1 signaling pathway.

Adiponectin/AdipoR1/APPL1 inhibits oxidative stress via $H O-1 / H M G B 1$. To ascertain whether adiponectin reduces oxidative stress in cardiomyocyte senescence through HO-1/ HMGB1 signaling, commercial kits were once again used to determine the ROS and MDA levels in D-gal-treated $\mathrm{H} 9 \mathrm{c} 2$ cells. In this experiment, $\mathrm{ZnPP}$ was used to treat the D-gal-treated cells overexpressing adiponectin. As shown in Fig. 5B and C, the relative ROS levels in D-gal-treated H9c2 cells were markedly decreased following adiponectin overexpression compared with the cells treated with D-gal alone; these levels were noticeably elevated following treatment with the HO-1 inhibitor. As shown in Fig. 5D, a similar trend was observed in MDA levels, indicating that adiponectin/AdipoR1/ APPL1 suppresses the release of oxidative stress in H9c2 cells treated with D-gal through HO-1/HMGB1 signaling.

\section{Discussion}

At 40 years of age, the remaining lifetime risk for developing certain types of CVD increases significantly in previously 


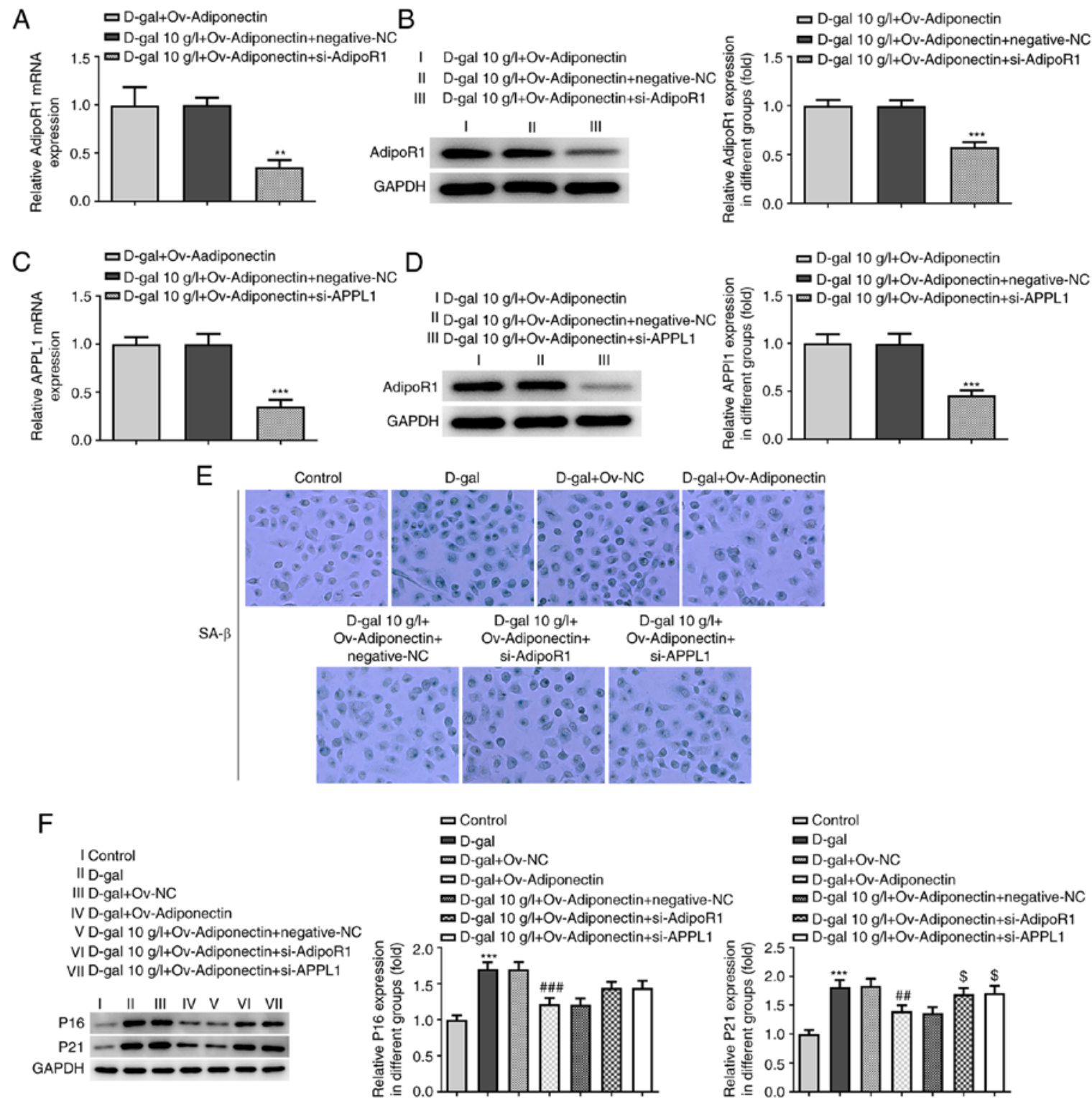

Figure 3. Adiponectin inhibits D-gal-induced cardiomyocyte senescence via AdipoR1/APPL1. (A and B) Relative expression levels of AdipoR1 in D-gal-treated $\mathrm{H} 9 \mathrm{c} 2$ cells with Ov-adiponectin before and after transfection with si-AdipoR1 and si-APPL were measured using RT-qPCR. ${ }^{* *} \mathrm{P}<0.01,{ }^{* * *} \mathrm{P}<0.001$ vs. D-gal + Ov-adiponectin. (C and D) Relative expression levels of APPL1 in D-gal-treated H9c2 cells with Ov-adiponectin before and after transfection with si-AdipoR1 and si-APPL were measured using RT-qPCR. ${ }^{* * *} \mathrm{P}<0.001$ vs. D-gal + Ov-adiponectin. (E) Cellular senescence in the different groups was detected using SA- $\beta$-gal staining. (F) Relative expression levels of p16 and p21 in the different groups were detected using western blot analysis. ${ }^{* * * *} \mathrm{P}<0.001 \mathrm{vs.}$ control; ${ }^{\# \#} \mathrm{P}<0.01,{ }^{\# \#} \mathrm{P}<0.001$ vs. D-gal + Ov-NC; ${ }^{\mathrm{P}} \mathrm{P}<0.05$ vs. D-gal + Ov-adiponectin + negative-NC. AdipoR1, adiponectin receptor 1; APPL1, adaptor protein phosphotyrosine interacting with PH domain and leucine zipper 1; Ov-, overexpression; RT-qPCR, reverse transcription-quantitative PCR; NC, negative control; si-, small interfering RNA; D-gal, D-galactose; SA- $\beta$-gal, senescence-associated $\beta$-galactose.

disease-free individuals; these CVDs can present in various forms, including chronic CVD, hypertension or heart failure (17). China, along with a number of other countries, has entered the stage of population aging $(18,19)$; thus, CVDs have become a leading cause of mortality worldwide, particularly among the elderly (20). It is also estimated that $\sim 20 \%$ of the world population will reach $\geq 65$ years of age by 2030 , which signifies not only an increase in the prevalence of CVDs, but also increased healthcare costs (2). Aging leads to structural and functional alterations in the cardiovascular system in an unfavorable manner (21). Cardiomyocyte senescence, as a part of the aging process, is directly associated with the dysfunction of myocardial tissues (22), the deterioration of which is often induced by oxidative stress (23). D-gal is commonly used in a number of rodent models of aging, including models of brain $(24,25)$, renal $(26,27)$ and heart $(28,29)$ aging. In the present study, H9c2 cells were treated with D-gal at various concentrations, and the concentration of $10 \mathrm{~g} / \mathrm{l}$ was selected to establish a cardiomyocyte senescence model to simulate the results of the animal experiment carried out in advance. It was found that the expression levels of adiponectin, AdipoR1 and APPL1 were decreased in the D-gal-treated cardiomyocytes, which was consistent with the results obtained in the animal experiments.

Jin et al (30) demonstrated that adiponectin, an endocrine factor secreted mainly by adipose tissues, reduced cellular senescence and led to the functional recovery of keratinocytes. Furthermore, adiponectin binds to its two receptors, AdipoR1 
A
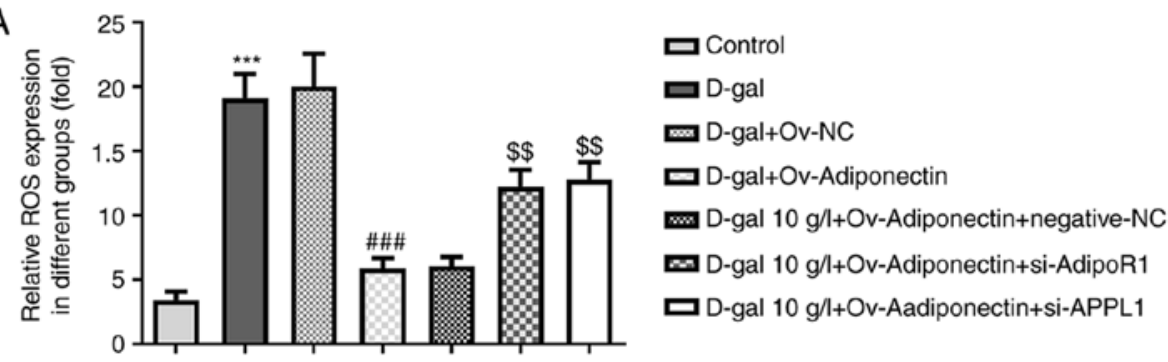

B
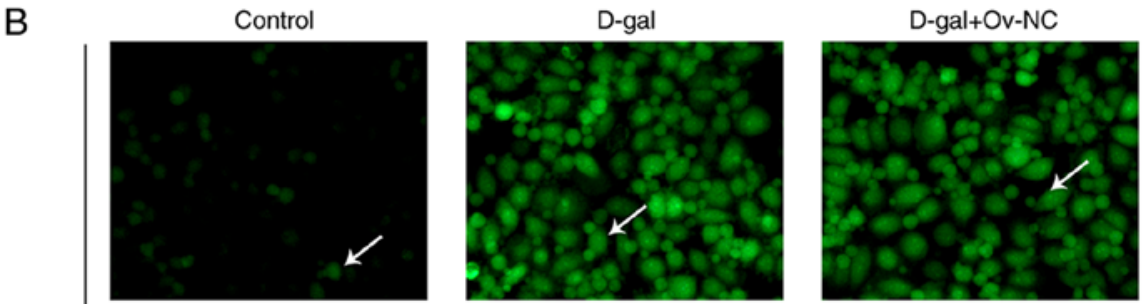

D-gal+Ov-Adiponectin
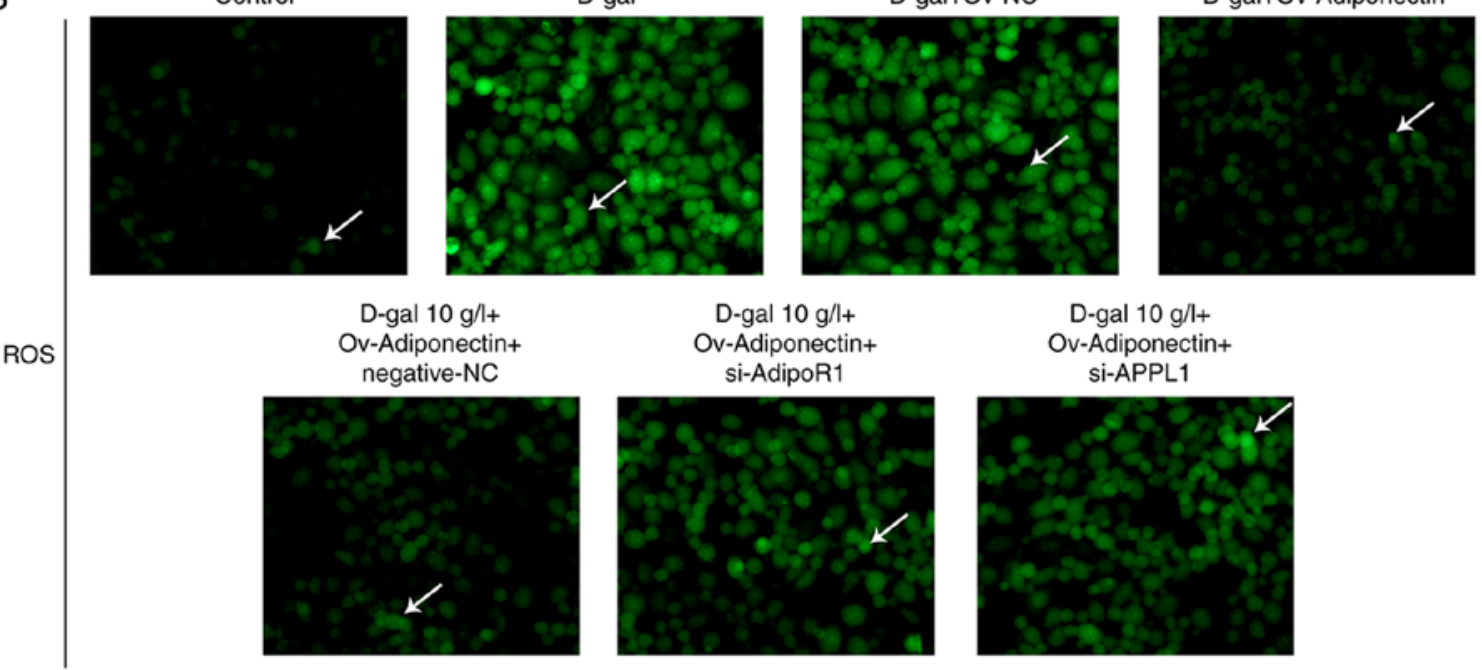

Ov-Adiponectin+

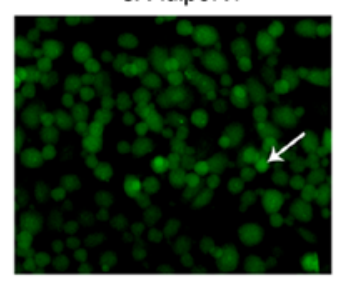

D-gal $10 \mathrm{~g} / \mathrm{l}+$

Ov-Adiponectin+ si-APPL1

C

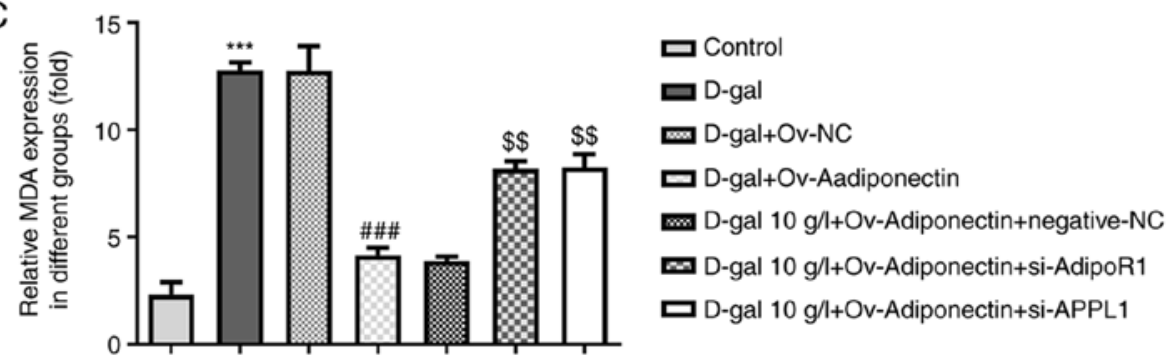

Figure 4. Adiponectin inhibits oxidative stress levels in D-gal-treated cardiomyocytes via AdipoR1/APPL1. (A) si-AdipoR1 and si-APPL1 were transfected into D-gal-treated H9c2 cells overexpressing adiponectin. The relative expression of ROS in the different groups was detected using a ROS Assay kit. ${ }^{* * *} \mathrm{P}<0.001$ vs. control; ${ }^{\# \#} \mathrm{P}<0.001$ vs. D-gal + Ov-NC; ${ }^{\$} \mathrm{P}<0.01$ vs. D-gal + Ov-adiponectin + negative-NC. (B) ROS production in the different groups was detected using the ROS Assay kit (magnification, $\mathrm{x} 200$ ). DCF fluorescence staining of the cells are indicated by arrows in the figure, the intensity of staining can reflect the level of ROS. (C) Relative MDA expression in the different groups was detected using the Lipid Peroxidation MDA Assay kit. ${ }^{* * *} \mathrm{P}<0.001$ vs. control; ${ }^{\# \#} \mathrm{P}<0.001$ vs. D-gal + Ov-NC; ${ }^{\$} \mathrm{P}<0.01 \mathrm{vs}$. D-gal + Ov-adiponectin + negative-NC. AdipoR1, adiponectin receptor 1 ; ROS, reactive oxygen species; MDA, malondialdehyde; APPL1, adaptor protein phosphotyrosine interacting with PH domain and leucine zipper 1; si-, small interfering RNA; Ov-, overexpression; D-gal, D-galactose; NC, negative control.

and R2, to initiate signaling transduction events, which are regulated by adaptor proteins, such as APPL1, that directly bind to the intracellular regions of AdipoRs (13). Visceral fat and adipocytes are risk factors for different forms of heart disease and heart failure (31). In the present study, an adiponectin overexpression plasmid was constructed and this was transfected into H9c2 cells treated with D-gal. The expression of AdipoR1 and APPL1 was then detected in the cells. A markedly higher expression of AdipoR1 and APPL1 was noted in the Ov-adiponectin group compared with the NC group, which indicated that adiponectin overexpression promoted AdipoR1 and APPL1 expression in D-gal-treated cardiomyocytes. For the purpose of identifying the mechanisms of adiponectin in cardiomyocyte senescence, si-AdipoR1 and si-APPL1 were transfected into D-gal-treated H9c2 cells overexpressing adiponectin to observe cellular senescence. The results revealed that adiponectin reduced senescent cells, potentially by regulating AdipoR1 and APPL1.

It has been demonstrated that increased levels of ROS are implicated in cell senescence-related pathogenesis (32). Adiponectin has previously been proven to exert protective effects against oxidative stress in a number of diseases, playing an antioxidant role in oxidative stress-associated skeletal muscle diseases (33) and reducing oxidative stress in diabetic nephropathy (34). Accordingly, the present study hypothesized that adiponectin may inhibit ROS production in D-gal-treated H9c2 cells, thus attenuating cardiomyocyte senescence. The examination of oxidative stress often involves measuring ROS levels, as well as MDA content, which is commonly used as a lipid peroxidation marker (35). Therefore, 
A

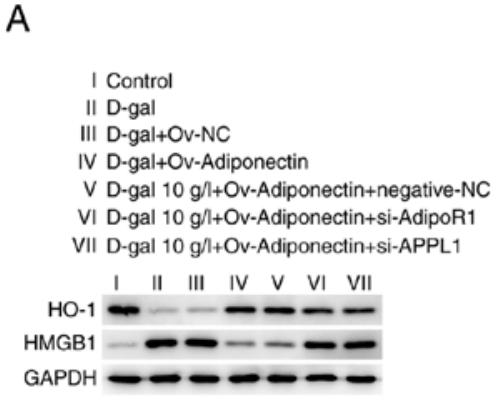

B

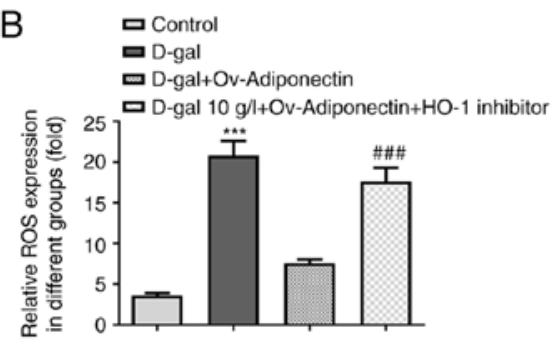

D

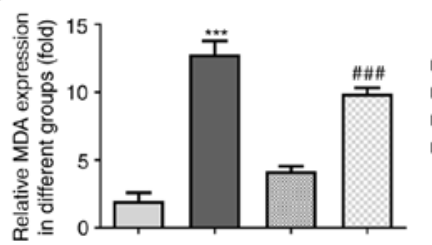

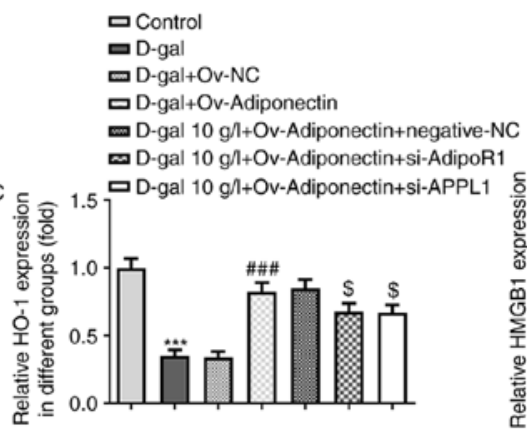

C Control

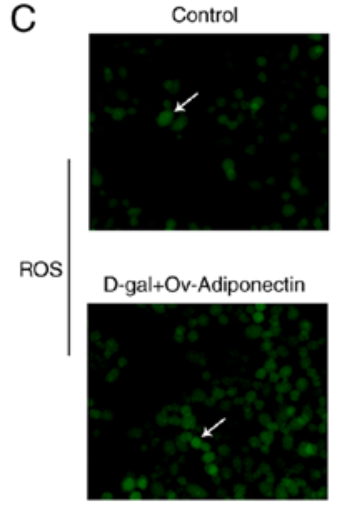

口 Control

口 D-gal

D-gal+Ov-Adiponectin

D D-gal 10 g/l+Ov-Adiponectin+HO-1 inhibitor

Figure 5. Adiponectin affects the release of HO-1/HMGB1 via AdipoR1/APPL1. (A) Relative expression of HO-1 in the different groups was detected using western blot analysis. Relative expression of HMGB1 in the different groups was detected using western blot analysis. ${ }^{* * *} \mathrm{P}<0.001 \mathrm{vs}$. control; ${ }^{\# \# \#} \mathrm{P}<0.001 \mathrm{vs}$. $\mathrm{D}$-gal + Ov-NC; ${ }^{\$} \mathrm{P}<0.05,{ }^{\$ \$ \$} \mathrm{P}<0.001$ vs. D-gal + Ov-adiponectin + negative-NC. Adiponectin/AdipoR1/APPL1 inhibits oxidative stress via HO-1/HMGB1. (B) $\mathrm{H} 9 \mathrm{C} 2$ cells overexpressing adiponectin were treated with HO-1 inhibitor.; the relative expression of ROS in the different groups was detected using a ROS Assay kit. ${ }^{* * *} \mathrm{P}<0.001$ vs. control; ${ }^{\# \# \#} \mathrm{P}<0.001$ vs. D-gal + Ov-adiponectin group. (C) ROS production in $\mathrm{H} 9 \mathrm{c} 2$ cells in the different groups was detected using a ROS Assay kit. DCF fluorescence staining of the cells is indicated by arrows in the figure, the intensity of staining can reflect the level of ROS (magnification, $\mathrm{x} 200$ ). (D) Relative expression of MDA in the different groups was detected using a Lipid Peroxidation MDA Assay kit. ${ }^{* * *} \mathrm{P}<0.001$ vs. control; ${ }^{\# \# "} \mathrm{P}<0.001$ vs. D-gal + Ov-adiponectin group. AdipoR1, adiponectin receptor 1; ROS, reactive oxygen species; HO-1, heme oxygenase 1; HMGB1, high mobility group box 1; APPL1, adaptor protein phosphotyrosine interacting with PH domain and leucine zipper 1; NC, negative control; Ov-, overexpression; si-, small interfering RNA; D-gal, D-galactose; MDA, malondialdehyde.

ROS production and the MDA content were detected in the present study, which verified that adiponectin inhibited oxidative stress levels in D-gal-induced cardiomyocyte senescence via AdipoR1/APPL1 signaling. Choubey et al (36) noted that adiponectin treatment improved the levels of increased oxidative stress during aging and thus improved testicular function during aging. Previous studies have also reported that some compounds (such as onion juice; vitamin $\mathrm{C}$ and hesperidin) show antioxidant properties (29-39). In addition, pretreatment with $n-3$ polyunsaturated fatty acids, such as fish oil and flaxseed oil, significantly inhibits myocardial injury (40).

A previous study found that adiponectin can activate HO-1 signaling, which thereby attenuated the production of ROS in HepG2 cells (41). Shan et al (42) revealed that HO-1 improves cardiac function and attenuates ischemic injury and aging-induced cardiomyocyte senescence. Additionally, it has been well-established that the expression of HMGB1, a proinflammatory adipocytokine, can be inhibited by both adiponectin and upstream HO-1. Shimizu et al (43) demonstrated that adiponectin can inhibit TNF- $\alpha$-induced HMGB1 secretion from 3T3-L1 adipocytes. Furthermore, Luo et al (44) observed that HO-1 can inhibit HMGB1 activity in lipopolysaccharide-induced acute lung injury in vitro. Based on the aforementioned evidence, the present study performed a series of experiments to examine the association between adiponectin, AdipoR1/APPL1 and HO-1/HMGB1 in D-gal-induced cardiomyocyte senescence. It was found that adiponectin overexpression elevated HO-1 expression levels and decreased HMGB1 expression levels in D-gal-treated H9c2 cells, which was consistent with the findings of previous studies. Moreover, in contrast to the NC group, HO-1 expression was decreased and HMGB1 expression was increased following transfection of the D-gal-treated $\mathrm{H} 9 \mathrm{c} 2$ cells overexpressing adiponectin with si-AdipoR1 and si-APPL1. It can be concluded from the results that adiponectin affects the release of HO-1/HMGB1 through AdipoR1/APPL1 signaling.

Furthermore, the present study further explored the inhibitory effects of adiponectin on the levels of oxidative stress in senescent cardiomyocytes, as well as the role of HO-1/HMGB1 in such a mechanism. In a previous study, Chen et al (45) reported that HO-1 overexpression reduced the production of mitochondrial oxidation to prevent myocardial 
hypoxia-reoxygenation injury in $\mathrm{H} 9 \mathrm{c} 2$ cells. Similarly, the present study used ZnPP to inhibit HO-1 activity, and detected the expression levels of ROS and MDA in H9c2 cells in different groups. The results demonstrated that while adiponectin overexpression notably decreased the oxidative stress levels, the inhibition of HO-1 restored the levels of oxidative stress to a large extent, suggesting that adiponectin/AdipoR1/ APPL1 inhibits oxidative stress through HO-1/HMGB1 signaling.

In conclusion, the present study provided evidence to suggest that adiponectin protected against cardiomyocyte senescence induced by D-gal via AdipoR1/APPL1 signaling, and that it attenuated oxidative stress in senescent $\mathrm{H} 9 \mathrm{c} 2$ cells by inhibiting the HO-1/HMGB1 signaling pathway. To the best of our knowledge, the present study was the first to elucidate the mechanisms of action of adiponectin in cardiomyocyte senescence induced by $\mathrm{D}$-gal and shed light on the application of adiponectin in the treatment of age-related CVDs. These results also provided viable targets and research directions for clinical myocardial senescence treatment. The majority of the experiments in the present study were conducted in vitro; thus, further in vivo experiments are required to examine the efficacy of adiponectin on cardiomyocyte senescence-associated CVDs in order to validate the current findings and to assist future clinical trials.

\section{Acknowledgements}

Not applicable.

\section{Funding}

The present study was supported by Medical Guidance (TCM) Science and Technology Support Project of Science and Technology Commission of Shanghai Municipality (grant no. 17401933700), the Scientific Research Project of Shanghai Municipal Health Commission (grant no. 201640039) and the Peak Plateau Subject of Shanghai University of Traditional Chinese Medicine (special project for clinical talents, grant no. 171319).

\section{Availability of data and materials}

The datasets used and/or analyzed during the current study are available from the corresponding author on reasonable request.

\section{Authors' contributions}

DL and RL contributed to conception and design of the study. DL, DL and JM performed the experiments and data collection. RL and JM contributed to analysis and interpretation of data. DL revised the manuscript critically for important intellectual content. DL and RL confirm the authenticity of all the raw data. All authors read and approved the final version of the manuscript.

\section{Ethics approval and consent to participate}

Ethical approval was obtained from The Shanghai Municipal Hospital of Traditional Chinese Medicine (approval no. dw2019018; Shanghai, China).

\section{Patient consent for publication}

Not applicable.

\section{Competing interests}

The authors declare that they have no competing interests.

\section{References}

1. Sander M, Oxlund B, Jespersen A, Krasnik A, Mortensen EL, Westendorp RG and Rasmussen LJ: The challenges of human population ageing. Age Ageing 44: 185-187, 2015.

2. Costantino S, Paneni F and Cosentino F: Ageing, metabolism and cardiovascular disease. J Physiol 594: 2061-2073, 2016.

3. Zhao D, Liu J, Wang M, Zhang X and Zhou M: Epidemiology of cardiovascular disease in China: Current features and implications. Nat Rev Cardiol 16: 203-212, 2019.

4. Liguori I, Russo G, Curcio F, Bulli G, Aran L, Della-Morte D, Gargiulo G, Testa G, Cacciatore F, Bonaduce D and Abete P: Oxidative stress, aging, and diseases. Clin Interv Aging 13: 757-772, 2018.

5. Martin-Fernandez B and Gredilla R: Mitochondria and oxidative stress in heart aging. Age (Dordr) 38: 225-238, 2016.

6. Wang LF, Cao Q, Wen K, Xiao YF, Chen TT, Guan XH, Liu Y, Zuo L, Qian YS, Deng KY and Xin HB: CD38 deficiency alleviates D-galactose-induced myocardial cell senescence through NAD+/Sirtl signaling pathway. Front Physiol 10: 1125, 2019.

7. Du H, Wang Y, Liu X, Wang S, Wu S, Yuan Z and Zhu X: miRNA-146a-5p mitigates stress-induced premature senescence of D-galactose-induced primary thymic stromal cells. Cytokine 137: 155314, 2021.

8. Sun K, Yang P, Zhao R, Bai Y and Guo Z: Matrine attenuates $\mathrm{D}$-galactose-induced aging-related behavior in mice via inhibition of cellular senescence and oxidative stress. Oxid Med Cell Longev 2018: 7108604, 2018.

9. Bo-Htay C, Palee S, Apaijai N, Chattipakorn SC and Chattipakorn N: Effects of d-galactose-induced ageing on the heart and its potential interventions. J Cell Mol Med 22: 1392-1410, 2018.

10. Frimat M, Daroux M, Litke R, Neviere R, Tessier FJ and Boulanger E: Kidney, heart and brain: Three organs targeted by ageing and glycation. Clin Sci (Lond) 131: 1069-1092, 2017.

11. Wang ZV and Scherer PE: Adiponectin, the past two decades. J Mol Cell Biol 8: 93-100, 2016.

12. Huang J, Hou B, Zhang S, Wang M, Lu X, Wang Q and Liu Y: The protective effect of adiponectin-transfected endothelial progenitor cells on cognitive function in D-galactose-induced aging rats. Neural Plast 2020: 1273198, 2020.

13. Fang $\mathrm{H}$ and Judd RL: Adiponectin regulation and function. Compr Physiol 8: 1031-1063, 2018.

14. Xu N, Zhang Y, Doycheva DM, Ding Y, Zhang Y, Tang J, Guo H and Zhang JH: Adiponectin attenuates neuronal apoptosis induced by hypoxia-ischemia via the activation of AdipoR1/APPL1/ LKB1/AMPK pathway in neonatal rats. Neuropharmacology 133: 415-428, 2018.

15. Livak KJ and Schmittgen TD: Analysis of relative gene expression data using real-time quantitative PCR and the 2(-Delta Delta C(T)) method. Methods 25: 402-408, 2001.

16. Sun Z, Wang F, Yang Y, Wang J, Sun S, Xia H and Yao S: Resolvin D1 attenuates ventilator-induced lung injury by

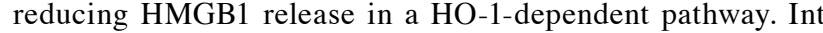
Immunopharmacol 75: 105825, 2019.

17. Lakatta EG: So! What's aging? Is cardiovascular aging a disease? J Mol Cell Cardiol 83: 1-13, 2015.

18. Bundy JD and He J: Hypertension and related cardiovascular disease burden in China. Ann Glob Health 82: 227-233, 2016

19. Li H, Jia J and Yang Z: Mini-mental state examination in elderly Chinese: A population-based normative study. J Alzheimers Dis 53: 487-496, 2016.

20. Evans MA, Sano S and Walsh K: Cardiovascular disease, aging, and clonal hematopoiesis. Annu Rev Pathol 15: 419-438, 2020.

21. Obas V and Vasan RS: The aging heart. Clin Sci (Lond) 132: 1367-1382, 2018.

22. Anderson R, Lagnado A, Maggiorani D, Walaszczyk A, Dookun E, Chapman J, Birch J, Salmonowicz H, Ogrodnik M, Jurk D, et al: Length-independent telomere damage drives post-mitotic cardiomyocyte senescence. EMBO J 38: e100492, 2019. 
23. Matsushima $\mathrm{S}$ and Sadoshima J: The role of sirtuins in cardiac disease. Am J Physiol Heart Circ Physiol 309: H1375-H1389, 2015.

24. Kou X, Liu X, Chen X, Li J, Yang X, Fan J, Yang Y and Chen N: Ampelopsin attenuates brain aging of D-gal-induced rats through miR-34a-mediated SIRT1/mTOR signal pathway. Oncotarget 7: 74484-74495, 2016.

25. Sadigh-Eteghad S, Majdi A, McCann SK, Mahmoudi J, Vafaee MS and Macleod MR: D-galactose-induced brain ageing model: A systematic review and meta-analysis on cognitive outcomes and oxidative stress indices. PLoS One 12: e0184122, 2017.

26. Liu B, Tu Y, He W, Liu Y, Wu W, Fang Q, Tang H, Tang R, Wan Z, Sun W and Wan Y: Hyperoside attenuates renal aging and injury induced by D-galactose via inhibiting AMPK-ULK1 signaling-mediated autophagy. Aging (Albany NY) 10: 4197-4212, 2018

27. El-Horany HE, Gaballah HH and Helal DS: Berberine ameliorates renal injury in a rat model of D-galactose-induced aging through a PTEN/Akt-dependent mechanism. Arch Physiol Biochem 126: 157-165, 2020

28. Dehghani A, Hafizibarjin Z, Najjari R, Kaseb F and Safari F: Resveratrol and 1,25-dihydroxyvitamin D co-administration protects the heart against D-galactose-induced aging in rats: Evaluation of serum and cardiac levels of klotho. Aging Clin Exp Res 31: 1195-1205, 2019.

29. Bei Y, Wu X, Cretoiu D, Shi J, Zhou Q, Lin S, Wang H, Cheng Y, Zhang $\mathrm{H}$, Xiao $\mathrm{J}$ and $\mathrm{Li} \mathrm{X}$ : miR-21 suppression prevents cardiac alterations induced by d-galactose and doxorubicin. J Mol Cell Cardiol 115: 130-141, 2018.

30. Jin T, Kim MJ, Heo WI, Park KY, Choi SY, Lee MK, Hong SP, Kim SJ, Im M, Moon NJ and Seo SJ: Adiponectin corrects premature cellular senescence and normalizes antimicrobial peptide levels in senescent keratinocytes. Biochem Biophys Res Commun 477: 678-684, 2016.

31. Gonzalez N, Moreno-Villegas Z, Gonzalez-Bris A, Egido J and Lorenzo O: Regulation of visceral and epicardial adipose tissue for preventing cardiovascular injuries associated to obesity and diabetes. Cardiovasc Diabetol 16: 44, 2017.

32. Davalli P, Mitic T, Caporali A, Lauriola A and D'Arca D: ROS, cell senescence, and novel molecular mechanisms in aging and age-related diseases. Oxid Med Cell Longev 2016: 3565127, 2016.

33. Ren Y, Li Y, Yan J, Ma M, Zhou D, Xue Z, Zhang Z, Liu H, Yang $\mathrm{H}$, Jia L, et al: Adiponectin modulates oxidative stress-induced mitophagy and protects $\mathrm{C} 2 \mathrm{C} 12$ myoblasts against apoptosis. Sci Rep 7: 3209, 2017.

34. Lee JY, Yang JW, Han BG, Choi SO and Kim JS: Adiponectin for the treatment of diabetic nephropathy. Korean J Intern Med 34: 480-491, 2019
35. Morales M and Munne-Bosch S: Malondialdehyde: Facts and artifacts. Plant Physiol 180: 1246-1250, 2019.

36. Choubey M, Ranjan A, Bora PS, Baltazar F, Martin LJ and Krishna A: Role of adiponectin as a modulator of testicular function during aging in mice. Biochim Biophys Acta Mol Basis Dis 1865: 413-427, 2019.

37. Shokoohi M, Madarek EOS, Khaki A, Shoorei H, Khaki AA, Soltani $\mathrm{M}$ and Ainehchi N: Investigating the effects of onion juice on male fertility factors and pregnancy rate after testicular torsion/detorsion by intrauterine insemination method. Int J Womens Health and Reprod Sci 6: 499-505, 2018.

38. Moghimian M, Soltani M, Abtahi H and Shokoohi M: Effect of vitamin $\mathrm{C}$ on tissue damage and oxidative stress following tunica vaginalis flap coverage after testicular torsion. J Pediatr Surg 52: 1651-1655, 2017.

39. Shokoohi M, Khaki A, Shoorei H, Khaki AA, Moghimian M and Abtahi-Eivary SH: Hesperidin attenuated apoptotic-related genes in testicle of a male rat model of varicocoele. Andrology 8: 249-258, 2020.

40. Ivary SHA, Jajarmy N, Shahri MK, Shokoohi M, Shoorei H, Ebadi A, Moghimian M and Sigaroodi F: Effect of fish and flaxseed oil supplementation on isoprenaline-induced myocardial infarction in rats: Inhibition of mitochondrial permeability transition pore opening. Crescent J Med Biol Sci 6: 158-163, 2019.

41. Shrestha A and Park PH: Globular adiponectin attenuates LPS-induced reactive oxygen species production in HepG2 cells via FoxO3A and HO-1 signaling. Life Sci 148: 71-79, 2016.

42. Shan H, Li T, Zhang L, Yang R, Li Y, Zhang M, Dong Y, Zhou Y, $\mathrm{Xu} \mathrm{C}$, Yang $\mathrm{B}$, et al: Heme oxygenase-1 prevents heart against myocardial infarction by attenuating ischemic injury-induced cardiomyocytes senescence. EBioMedicine 39: 59-68, 2019.

43. Shimizu T, Yamakuchi M, Biswas KK, Aryal B, Yamada S, Hashiguchi T and Maruyama I: HMGB1 is secreted by 3T3-L1 adipocytes through JNK signaling and the secretion is partially inhibited by adiponectin. Obesity (Silver Spring) 24: 1913-1921, 2016.

44. Luo M, Hong XQ, Zhu H, Li G and Tang L: The HO-1 signal prevents HMGB1-mediated activation of NLRP3 inflammasomes in lipopolysaccharide-induced acute lung injury in vitro. J Surg Res 247: 335-343, 2020.

45. Chen D, Jin Z, Zhang J, Jiang L, Chen K, He X, Song Y, Ke J and Wang Y: HO-1 protects against hypoxia/reoxygenation-induced mitochondrial dysfunction in $\mathrm{H} 9 \mathrm{c} 2$ cardiomyocytes. PLoS One 11: e0153587, 2016.

This work is licensed under a Creative Commons Attribution-NonCommercial-NoDerivatives 4.0 International (CC BY-NC-ND 4.0) License. 